\title{
DIVERSIFICAÇÃO TECNOLÓGICA EM AMBIENTES EM TRANSFORMAÇÃO: UM ESTUDO A PARTIR DE TECNOLOGIAS DE MITIGAÇÃO DAS MUDANÇAS CLIMÁTICAS
}

Paulo Henrique Assis Feitosa ${ }^{1}$

\section{Resumo}

Este artigo analisa a diversificação das firmas em tecnologias de mitigação das mudanças climáticas e os efeitos das alterações nos níveis de oportunidades tecnológicas. A partir da constituição de um banco de dados original com patentes relacionadas a tecnologias de mitigação das mudanças climáticas, o artigo examina 120 grupos de tecnologias em todo mundo e o portfólio de patentes de 436 firmas, entre os anos 1990-2009. As estimativas econométricas evidenciam o efeito que o ambiente tecnológico exerce sobre a atividade inovadora de firmas de diferentes estruturas, localização e atividades econômicas. Os resultados sugerem uma relação não linear em formato de "U" invertido entre o nível de oportunidade tecnológica e o grau de diversificação das firmas. Em ambientes tecnológicos marcados por rápida transformação, as firmas adotam estratégias de especialização, direcionando suas atividades para o reforço de áreas tecnológicas existentes. No mesmo sentido, maiores graus de diversificação são observados em firmas com grande porte e maior capacidade acumular conhecimentos.

Palavras-chave: Diversificação tecnológica; oportunidades tecnológicas; mudanças climáticas.

\begin{abstract}
This article analyzes the diversification of firms in climate change-mitigation technologies and the effects of changes in the levels of technological opportunities. From the constitution of a unique database of patents related to mitigation of climate change technologies, the article examines 120 technology groups worldwide and the patent portfolio of 436 firms, between the years 1990-2009. Econometric estimates reveal the effect that technological environment carries on the innovative activity of firms of different structures, location and economic activities. The results suggest a non-linear relationship (inverse-U-shape) between the level of technological opportunity and the degree of diversification of firms. In technological environments characterized by rapid change, firms adopt specialization strategies, directing its activities to reinforcement existing technological areas. Similarly, large firms and firms with greater capacity to accumulate knowledge have higher levels of technological diversification.
\end{abstract}

Keywords: Technological diversification; technological opportunities; climate change.

Área ENEI 5: Mudanças técnicas, organizações e instituições; transferência de tecnologia e processos de aprendizado; competências e capacitações das empresas; inovação, competências e competitividade; indicadores de ciência, tecnologia, inovação.

Classificações JEL: L25, O31, O34, Q54, Q55.

\footnotetext{
${ }^{1}$ Doutorando em Ciência Econômica do Instituto de Economia da Universidade Estadual de Campinas (Unicamp).

Email: paulohenriquefeitosa@gmail.com.
} 


\section{Introdução}

A diversificação está no cerne da evolução das firmas modernas, que a utilizam como estratégia de crescimento de longo prazo via expansão para novos mercados geográficos e de produtos. Nessa estratégia, as firmas buscam constantemente capturar novos investimentos lucrativos, motivadas pela possibilidade de eliminar as restrições à sua expansão, impostas pela demanda potencial dos produtos que compõem o seu portfólio.

A diversificação é estudada de forma ampla pela literatura e tradicionalmente relacionada com questões como a redução de custos e riscos, e a exploração de economias de escala e escopo. Mais recentemente, diante do reconhecimento do crescente papel do conhecimento científico e tecnológico para explicar o crescimento econômico no mundo moderno, estudiosos se dedicam a compreender a dimensão "tecnológica" da diversificação corporativa. Nessa abordagem, a diversificação tecnológica é percebida como uma forma pela qual as firmas criam, ampliam ou modificam a sua "coleção de recursos produtivos", que são específicos do contexto de cada firma e fortemente relacionados com suas competências e rotinas internas. A construção dessas capacitações é resultado de processos internos de aprendizado que são experimentados de forma evolucionária em ambientes de seleção.

A revisão da literatura identifica um amplo conjunto de análises empíricas sobre as causas e os efeitos da diversificação, a destacar a ênfase nas relações de causalidade entre o nível de diversificação tecnológica das firmas e seu desempenho inovativo, financeiro e produtivo. Como resultado, enquanto as relações que envolvem variáveis internas à firma foram preferidas e extensamente estudadas, as pesquisas que incluem variáveis que controlam os efeitos externos à firma no processo de diversificação tecnológica são mais escassas na literatura. As exceções a essa hegemonia são os estudos que consideram o papel das oportunidades externas à firma como elemento central do seu desempenho e evolução. Esses resultados mostram que o perfil tecnológico das grandes firmas é relativamente estável ao longo do tempo e que mudanças ocorrem em reposta ao surgimento de oportunidades (PATEL; PAVITT, 1997). Desta forma, o alcance das competências dominadas por essas firmas cresce ao longo do tempo na medida em que oportunidades tecnológicas emergem (PAVITT, 1998).

Entretanto, ainda que se reconheça a importância dos desenvolvimentos teóricos empreendidos, a influência das modificações do ambiente externo no processo de diversificação tecnológica das firmas é um fenômeno que precisa ser mais bem compreendido pela literatura. Estudos têm sugerido uma relação não-linear com formato de "U" invertido entre diversificação tecnológica e oportunidades ou desempenho tecnológico, mas são restritos a localização, tamanho e setores econômicos das firmas (CHANG et al., 2014; CHEN; SHIH; CHANG, 2012; CORRADINI; DEMIREL; BATTISTI, 2016; LETEN; BELDERBOS; VAN LOOY, 2007). Desta maneira, percebe-se uma lacuna na compreensão de como as firmas, de forma mais generalizada, adotam a diversificação em resposta a rápidas modificações nos níveis de oportunidades tecnológicas.

Dentre as transformações em curso e passíveis de análise, as mudanças climáticas constituem um importante caso a ser estudado. A mitigação das emissões de gases de efeito estufa (GEE) é um desafio global e a sua solução envolve questões amplas como maior nível de conscientização ambiental da sociedade, regulações, incentivos e políticas de Estado, bem como o reposicionamento das estratégias tecnológicas das firmas de todos os setores e tamanhos. O problema climático exige a reestruturação de ativos tecnológicos constituídos por firmas ao longo de décadas, e modificam os níveis de oportunidades tecnológicas relacionadas à manufatura, meios de distribuição, redes de serviços e tecnologias complementares. 
O presente artigo busca preencher essa lacuna e tem como objetivo investigar se a diversificação das firmas em tecnologias de mitigação das mudanças climáticas é afetada por alterações nas condições de oportunidades tecnológicas. Nesse sentido, tem como finalidade examinar os determinantes da diversificação tecnológica das firmas nesses campos técnicos, com ênfase no papel exercido pelas mudanças nos níveis de oportunidade tecnológica.

Para tanto, foi constituído um banco de dados original com informações de documentos de patentes que possuem campos técnicos (IPC) classificados pela Organização Mundial de Propriedade Intelectual (OMPI) como Tecnologias Ambientalmente Saudáveis (TAS), conforme listado pela Convenção-Quadro das Nações Unidas sobre Mudança do Clima (CQNUMC). A partir da criação de variáveis e do tratamento econométrico, é realizada uma análise de 120 grupos de tecnologias em todo mundo e a evolução do portfólio de patentes de 436 firmas, entre os anos 1990-2009.

O artigo está organizado em cinco seções, além desta introdução. A segunda seção discute os aspectos teóricos e conceituais das oportunidades e diversificação tecnológica. A terceira apresenta a descrição da base de dados construída. A especificação do modelo e suas variáveis são expostas na quarta seção. Os resultados empíricos são apresentados na seção 5 e a última seção apresenta as considerações finais.

\section{Mudanças climáticas, oportunidades e diversificação tecnológica \\ 2.1 Conhecimento organizacional e diversificação tecnológica}

A diversificação corporativa é um fenômeno onipresente no mundo econômico contemporâneo e está intimamente relacionado com as mudanças nas condições de oportunidade das firmas. Esse fenômeno é observado pelo grau de dispersão das atividades das firmas em algum intervalo de tempo, e resulta da busca contínua pelo uso eficiente de recursos produtivos por meio da captura de oportunidades externas.

As investigações sobre a existência de algo inerente à natureza das firmas, capaz de promover ou limitar o seu crescimento, revelou o papel central exercido pela utilização de recursos produtivos internos às firmas. A ênfase nos recursos internos está associada à ideia de que o entorno relevante para as firmas é menos imutável do que se pressupõe, e passível de ser transformado a serviço de seus propósitos (PENROSE; PITELIS, 2009). Em outras palavras, o conjunto de oportunidades para investimento e crescimento percebidas por empresários e administradores é específico de cada firma e varia de acordo como os recursos que são utilizados ou ampliados por meio da aquisição externa.

Se comparada com abordagens convencionais da firma, a perspectiva baseada em recursos tem como vantagem a possibilidade de acomodação de uma das principais características das firmas modernas que é o seu caráter de multiproduto. Entretanto, resta compreender as condições de oportunidade que induzem as firmas a investir em novas linhas de produto mais do que investem em linhas tradicionais de negócios. Em outras palavras, as condições que levam os fabricantes de aeronaves a produzir mísseis e veículos espaciais, ou petroleiras a buscar a produção de energia a partir de fontes alternativas (TEECE, 1980, 1982).

Nesse sentido, as firmas diversificam suas atividades em situações em que existe a oportunidade de capturar economias de escala, ou seja, quando a produção de dois ou mais produtos depende de uma base de knowhow de propriedade comum e constante troca, e quando ativos especializados e indivisíveis são um insumo comum na produção de dois ou mais produtos (TEECE, 1980). Nessas condições, uma organização multiproduto tende a ser um modo eficiente de organização.

Existe um importante esforço de investigação das repercussões da diversificação no crescimento, estrutura e limites da firma. Se por um lado, parte influente dessa literatura tem posicionado questões como escolhas, contratos e trocas em ambientes de mercado como elementos centrais de análise, outra parte desse esforço 
tem revelado que essas interações envolvem de forma crescente a variável "conhecimento organizacional" e exige um arcabouço teórico em que o tratamento da firma inclua adequadamente seus mecanismos de criação e captura de conhecimento (HODGSON, 1998).

De acordo com as abordagens baseadas em capacitações, as firmas são percebidas como agrupamentos de recursos intangíveis e rotinas organizacionais de difícil imitação e transferência. Esse conhecimento tem como característica ser altamente específico, diferenciado e persistente ao longo do tempo, e sua acumulação é um processo marcado por path dependence, tacitividade, idiossincrasias e complexas interações (DOSI; NELSON; WINTER, 2001; NELSON; WINTER, 1982).

Essa abordagem é fundamentada na distinção conceitual entre elementos como os "artefatos", entendidos como os produtos e serviços que as firmas desenvolvem e comercializam, os "conhecimentos tecnológicos" subjacentes a essa capacidade e que são específicos de cada firma, e as "formas e procedimentos organizacionais" que elas utilizam para transformar conhecimentos em produtos e serviços ${ }^{2}$ (PAVITT, 1998).

Como a produção de novos artefatos requer a combinação e integração de uma ampla variedade de conhecimentos tecnológicos, as evidências mostram que a diversificação tecnológica das firmas é geralmente maior do que a diversificação de produtos. Diante da constante incorporação de novas tecnologias, grande parte das firmas pode ser considerada multitecnológica ainda que seja especializada em apenas uma linha de negócios. Esse diagnóstico é corroborado por um fato comum na evolução das indústrias em todo mundo, que é a prevalência de firmas "tecnologicamente diversificadas", ou seja, as firmas que inovam e controlam cada vez mais diferentes campos tecnológicos. Essas firmas também registram crescentes níveis de investimento em pesquisa e desenvolvimento, forte articulação externa para o desenvolvimento de novas tecnologias e a busca por oportunidades para ingressar em novos mercados a partir do relacionamento de tecnologias (GRANSTRAND; PATEL; PAVITT, 1997; PATEL; PAVITT, 1997).

As evidências também revelam que o perfil do conhecimento tecnológico das firmas é bastante estável ao longo do tempo ${ }^{3}$. A direção futura de acumulação tecnológica é restringida pela base de conhecimento mantido pelas firmas no presente, diante do forte componente path dependence desse processo. Esse conhecimento determina o que a firma pesquisa e tem capacidade de produzir, dada a existência de limites cognitivos. Além de estável, esse perfil é bastante similar para a maioria das firmas do mesmo segmento industrial, mas fortemente diferenciado entre aquelas que compõem diferentes segmentos. Sabe-se que, para as firmas individuais, a diversidade tecnológica garante a base para aperfeiçoar seus produtos. Para a economia, como um todo, a diversidade entre as firmas possibilita uma exploração mais completa de mercados de produtos (PATEL; PAVITT, 1997; PAVITT, 1998).

O desenvolvimento desses conhecimentos tecnológicos pela firma ocorre por meio de um processo de aprendizagem gradual que restringe sua atividade de pesquisa a áreas próximas àquelas nas quais ela já possui domínio. A justificativa principal é o custo para se dominar conhecimentos especializados no mundo

\footnotetext{
${ }^{2}$ Esses elementos são necessários, mas nenhum é suficiente por si só. Uma repercussão desse fato sobre estudos empíricos em geral é que um corpo de conhecimento não pode ser facilmente associado a uma específica classe de produtos, como propõe (SCHERER, 1982).

${ }^{3}$ Um estudo com um conjunto de 440 firmas revela que mais de $90 \%$ possuem perfil tecnológico similar quando comparados os períodos 1969-1974 e 1985-1990 (PATEL; PAVITT, 1997).
} 
contemporâneo, principalmente em grandes firmas, que possuem conhecimentos em um vasto número de campos tecnológicos (CANTWELL; FAI, 1999; PAVITT, 1998).

As análises sobre a natureza da diversificação tecnológica revelam um fenômeno multifacetado e determinado por questões como: o aumento da complexidade dos artefatos e conhecimentos tecnológicos; o acúmulo de conhecimentos fora das competências centrais da firma para dominar as tecnologias subjacentes a seus artefatos; e a base de conhecimentos exigida para aumentar a efetividade da apropriação dos retornos dos esforços de inovação e o lucro esperado (LETEN; BELDERBOS; VAN LOOY, 2007; TORRISI; GRANSTRAND, 2004). Essas análises também mostram que a diversificação não ocorre aleatoriamente e possuem certa intencionalidade, em outras palavras, as firmas possuem coerência nas atividades produtivas e tecnológicas que desempenham (SCOTT, 1993; TEECE et al., 1994). Da mesma forma, o inter-relacionamento de conhecimento é um fator-chave na explicação da diversificação tecnológica das firmas (BRESCHI; LISSONI; MALERBA, 2003).

\subsection{Mudanças no ambiente tecnológico e no nível de oportunidades}

No presente artigo, é especialmente relevante a compreensão dos efeitos das mudanças nas condições do ambiente tecnológico, mais especificamente nas oportunidades tecnológicas que são um elemento chave. Partindo da literatura existente, percebe-se que a influência do ambiente tecnológico em rápida transformação no processo de diversificação das firmas é um efeito que precisa ser mais bem compreendido. Nesse sentido, o contexto das mudanças climáticas constitui um importante caso de estudo. A mitigação das emissões de emissões de gases de efeito estufa (GEE) é um problema de escala internacional e exige o reposicionamento de estratégia das firmas de todos os tipos e tamanho, além da reestruturação de ativos tecnológicos constituídos por firmas ao longo de décadas (GEELS, 2014). A exploração dessas tecnologias tende a ocorrer internacionalmente e criam um amplo conjunto de oportunidades relacionadas à manufatura, meios de distribuição, redes de serviços e tecnologias complementares. Segundo a OCDE (2014), iluminação, energia elétrica, veículos híbridos e elétricos, geração de energia, baterias e motores são percebidos como as áreas de desenvolvimento mais acelerado de novas tecnologias.

De modo geral a oportunidade tecnológica reflete a facilidade para se inovar a partir de um determinado volume de recursos em pesquisa e pode ser classificada de acordo com seu nível e pervasividade (MALERBA; ORSENIGO, 1993). Altos níveis de oportunidade constituem um forte incentivo para realização de atividades inovadoras, ainda que sejam diferenciadas entre setores e tecnologias. Em alguns setores industriais essas oportunidades são relacionadas a P\&D em equipamentos e instrumentação, enquanto que em outras as fontes externas de conhecimento como fornecedores e clientes desempenham o papel principal. As condições de oportunidade são dinâmicas por natureza e não se mantém constantes ou exógenas, pois são geradas e recriadas pela atividade inovativa das firmas. Já a pervasividade das oportunidades é determinada pela capacidade de um novo conhecimento ser aplicado em uma variedade de produtos e mercados.

Pode-se distinguir três fontes principais de oportunidades tecnológicas nas indústrias (KLEVORICK et al., 1995). A primeira e mais importante advém dos avanços na compreensão científica que fundamenta toda a atividade de pesquisa e desenvolvimento das firmas. Os avanços na ciência básica têm impulsionado as taxas de avanço tecnológico em uma ampla gama de setores e têm sido um requisito fundamental para a maioria do progresso tecnológico realizado desde o último século.

A segunda decorre do progresso técnico que tem origem em outras indústrias, ou seja, das oportunidades tecnológicas que surgem dos avanços tecnológicos no restante da economia. Um exemplo é o surgimento de combustíveis alternativos que geram oportunidades tecnológicas para empresas do setor de energia, mas 
também para empresas aéreas, do setor químico e uma ampla cadeia produtiva que pode ser potencialmente favorecida pelo uso desse combustível ${ }^{4}$.

Por fim, uma terceira fonte provém de retornos positivos (feedback). Em muitos setores industriais a pesquisa que é realizada no presente gera novos conhecimentos e aumenta as oportunidades tecnológicas futuras. Do mesmo modo, o que uma firma aprende com a sua atividade de P\&D pode ser reforçado por feedback de outras empresas envolvidas nos novos produtos ou processos.

A habilidade das firmas em integrar, construir e configurar suas competências para responder a rápidas mudanças no ambiente depende das suas capacitações dinâmicas (TEECE; PISANO; SHUEN, 1997). A capacitação dinâmica de uma firma é entendida como a sua capacidade de, propositadamente, criar, expandir ou modificar a sua base de recursos. Como essa capacitação é dependente do contexto no qual as firmas estão inseridas, aquelas que apresentem a melhor aptidão evolutiva podem sobreviver e crescer em seus mercados (HELFAT et al., 2007). Nesse sentido, toda firma diversificada é o resultado da constituição de capacitações dinâmicas para responder a mudanças nas oportunidades tecnológicas dos seus contextos de inserção.

O perfil tecnológico das firmas se modifica lentamente ao longo do tempo, em resposta a mudanças em oportunidades tecnológicas. Como resultado, em áreas com altas oportunidades tecnológicas as firmas tendem a se transformar em multiproduto bem como multitecnologia (PAVITT, 1998). O dinamismo ambiental é um fator que limita as vantagens associadas com competências específicas às firmas (LIN; CHANG, 2015). Os diferentes níveis de dinamismo do ambiente, que se aplicam em diferentes contextos, tornam as competências tecnológicas obsoletas mais rapidamente em alguns setores em detrimento de outros.

\section{Descrição da base de dados}

A base de dados utilizada nesse artigo tem como fonte de informação o Banco Mundial de Estatísticas de Patentes (PATSTAT) do European Patent Office (EPO), na sua edição de abril de 2012. Esse banco abrange mais de 80 escritórios de propriedade intelectual em todo mundo e contém mais de 70 milhões de documentos de patentes. Adicionalmente, o artigo também utiliza o banco de dados comercial ORBIS da Bureau Van Dijck (BvDEP), que contém informações administrativas de 120 milhões de empresas ou registros de negócios ao redor do mundo (em outubro de 2015).

Para a construção da base de dados utilizada nesse trabalho foi realizado um processo de extração e tratamento das informações em três etapas principais. A primeira etapa consiste na seleção das "patentes verdes". A partir do banco de dados do EPO/PATSTAT foram selecionados todos os depósitos de patentes que possuem um ou mais campos técnicos (IPCs) classificados no Green Inventory como Tecnologias Ambientalmente Saudáveis (TAS). Partindo de um universo de aproximadamente 73 milhões de patentes em todo mundo, foram selecionados 5.297.210 depósitos aqui denominadas "patentes verdes"5. Um relativo ineditismo desse resultado é a abrangência dos escritórios analisados, que atende a perspectiva global

\footnotetext{
${ }^{4}$ Outra importante fonte externa de oportunidade tecnológica advém do conhecimento produzido por universidades, institutos públicos e privados de pesquisa, associações técnicas e profissionais e inventores independentes.

${ }^{5}$ No contexto do presente trabalho, uma "patente verde" é determinada apenas pelo critério de possuir algum IPC identificado pelo Green Inventory como TAS. Já nos escritórios de propriedade intelectual o processo de determinação de uma patente verde é mais complexo pois envolve o julgamento de um especialista em cada caso.
} 
proposta pelo trabalho. Ainda nessa fase, foram selecionadas apenas as patentes que possuem prioridade resultando em 2.535.867 depósitos.

Partindo apenas das patentes que possuíam alguma prioridade, a etapa 2 identifica 764.668 prioridades para a aplicação de dois filtros. O primeiro filtro faz recorte temporal de vinte anos (1990-2009), tendo como base a atualidade máxima permitida pela edição de abril de 2012 do EPO/PATSTAT 6 . Essa proposta temporal considera a tendência da literatura que trata o processo de negociação e ratificação desse tratado internacional de compromissos de redução de emissões de gases de efeito estufa (GEE), ocorrido entre 1997 e 1999, como um marco analítico de diversas transformações relacionadas às mudanças climáticas. Esse recorte foi distribuído em períodos de cinco anos e se justifica por representar uma "janela temporal" adequada para avaliar o impacto tecnológico (HENDERSON; COCKBURN, 1996; NOOTEBOOM et al., 2007; PODOLNY; STUART, 1995), além de estar relacionado ao tempo médio de exame dos pedidos de patentes em escritórios em todo o mundo (DE RASSENFOSSE; PALANGKARAYA; WEBSTER, 2016).

O segundo filtro seleciona as firmas que no período de cinco anos, depositaram em média mais do que dez patentes. Esse critério é relevante para que a amostra revele apenas os depositantes que possuem uma sistemática e coerente atividade inovativa nessas tecnologias. Nessa etapa também foram excluídos inventores individuais, universidade e institutos de pesquisa.

$\mathrm{Na}$ etapa 3, a aplicação desses filtros resultou em 95.014 depósitos realizados por 436 firmas em 37 escritórios em todo mundo. Por fim, associa-se as informações dos primeiros depositantes de todas as patentes com informações da base de dados do Orbis. Essa tarefa tem como objetivo obter informações adicionais das firmas, principalmente o seu tamanho. O diagrama de fluxo que sintetiza o processo de extração e o tratamento das informações nas três etapas é apresentado na Figura 1.

Figura 1 - Diagrama de fluxo do processo de extração e tratamento dos dados

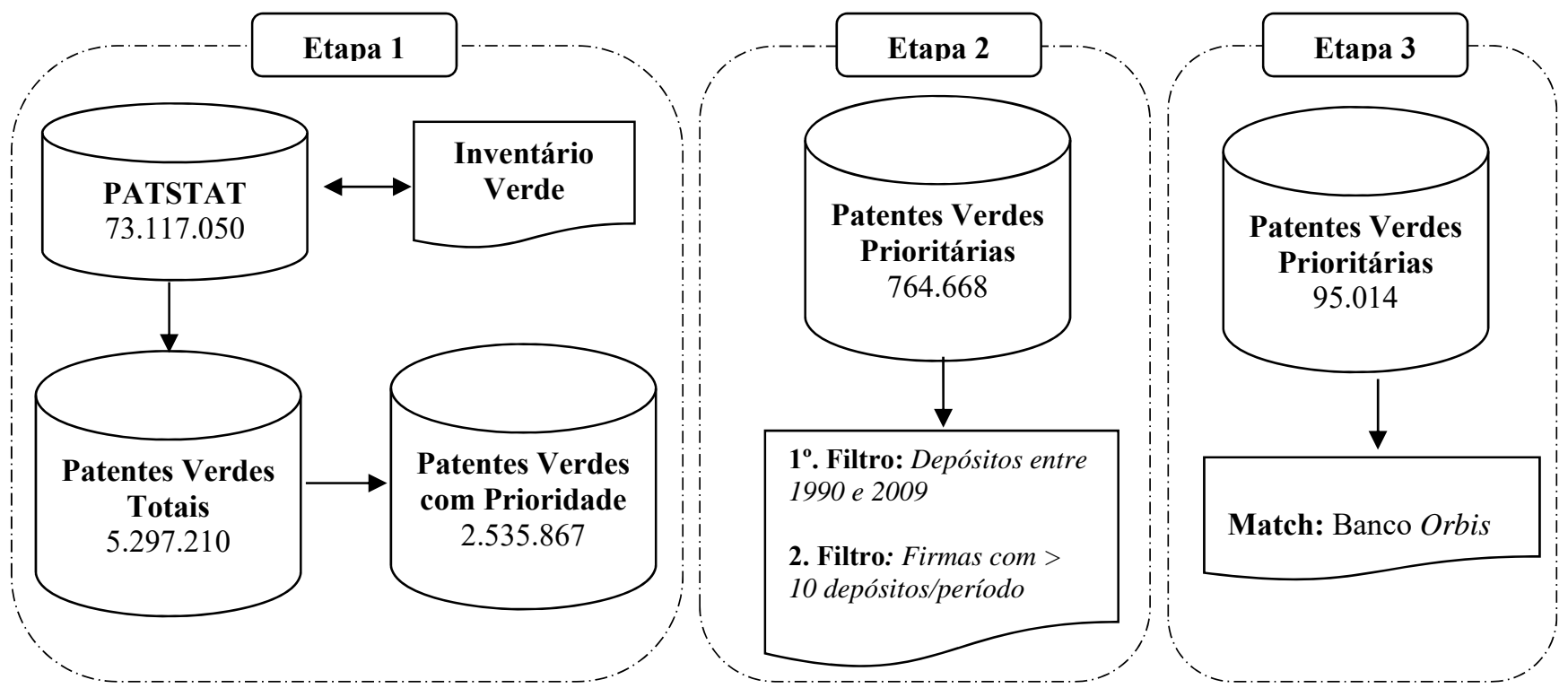

\footnotetext{
${ }^{6}$ Os documentos de referência para produção de estatística de patentes sugerem um mínimo de três anos entre sistematização de dados dos escritórios e produção de indicadores representativos.
} 


\section{Especificação do modelo \\ 4.1 Variável dependente}

A diversificação pode ser operacionalmente definida como o processo no qual uma gama de algo "A", associado a alguma unidade organizacional "B", tem como resultado um maior nível da amplitude ou extensão de "A". Nesses termos, "A" se refere a um conjunto de portfólios de produtos, serviços ou mercados, ou recursos como tecnologias, competências ou habilidades profissionais, enquanto a unidade "B" pode ser uma firma, indústria, país ou região, um indivíduo, bem como coletivo de indivíduos. Os elementos do conjunto " $A$ " são classificados ou categorizados de acordo com alguma taxonomia (tipologia, decomposição e desagregação) e o aumento da sua extensão significa, a partir de um ponto no tempo para outro, incluir elementos de um novo tipo (classe, categoria), não abrangido no ponto anterior do tempo (CANTWELL; GAMBARDELLA; GRANSTRAND, 2004; TORRISI; GRANSTRAND, 2004).

As medidas de diversificação foram extensamente utilizadas na literatura, mas com diferentes ênfases nas áreas de economia e negócios. No trabalho de Gort (1962) a diversificação é definida como "um aumento na heterogeneidade de produtos do ponto de vista do número de mercados servidos por este produto". Segundo o autor, dois produtos são vendidos em mercados distintos se suas elasticidades cruzadas da demanda são baixas e se, no curto prazo, os recursos empregados na produção e distribuição de um não pode ser deslocado para o outro. Berry (1971) definiu diversificação como o número de indústrias no qual uma firma é ativa. Para Kamie e Schwartz (1975) diversificação é a medida que as firmas classificadas em uma determinada indústria produzem bens classificados como pertencentes a outras indústrias. Um elemento incomum nessas definições é o fato de as fronteiras da indústria e dos mercados serem conhecidas previamente.

Em contraste, alguns autores também utilizaram o conceito de "negócios" ao invés de "indústrias" para definir a diversificação. Ansoff (1957) definiu a diversificação como o processo de entrada em uma nova área de mercado e/ou uma nova área de produtos que são novos para a empresa, mas não necessariamente novos para os mercados. Para Pitts e Hopkins (1982), diversificação é definida como a medida em que firmas operam em diferentes negócios simultaneamente. Ramanujam e Varadarajan (1989) definem diversificação como a entrada de uma firma ou unidade de negócios em novas linhas de atividade. Essa entrada implica em mudanças no sistema e estrutura administrativa e outros processos gerenciais, já que as ampliações nas linhas de produto sem implicações organizacionais não qualificam essas mudanças como uma diversificação nos negócios.

A definição exata de diversificação depende do agrupamento de produtos ou tecnologias que seja relevante para a análise em questão. Para determinados fins uma firma que produz apenas sapatos poderá ser considerada "não diversificada", enquanto que para outros propósitos, uma firma que produz todos os tamanhos e variedades de sapatos para todas as idades e sexos pode ser considerada diversificada. Consequentemente, não é possível nem desejável tentar estabelecer qualquer significado absoluto para esses termos, pois qualquer análise comparativa da "abrangência de diversificação", ou de estudos estatísticos do número de diferentes "produtos" por firmas terá sua utilidade limitada se não conhecermos as informações sobre as firmas e seus produtos (PENROSE; PITELIS, 2009).

Tradicionalmente diversificação refere-se à extensão e à distribuição dos resultados da empresa, classificada em termos de produtos, mercados ou negócios. A "diversificação dos produtos" é alcançada com diferentes "diversificações de insumos" ou ainda com a diversificação de recursos. No presente trabalho, esses recursos são classificados não apenas em termos de matérias primas, capital físico e financeiro, mas principalmente em termos de tecnologias, conhecimento, competências, propriedade intelectual e outros ativos intangíveis. As tecnologias são tomadas para significar artefatos de conhecimento 
técnico, o que torna a diversificação tecnológica um caso especial de diversificação do conhecimento, ou ainda diversificação de competências (TORRISI; GRANSTRAND, 2004).

No presente trabalho, para medir a diversificação tecnológica, adota-se um indicador empregado nos principais estudos empíricos para estimar o efeito de diversificação na atividade tecnológica (CORRADINI; BATTISTI; DEMIRELY, 2016; GARCIA-VEGA, 2006; LETEN; BELDERBOS; VAN LOOY, 2007). Esse indicador é estimado pelo inverso do índice Herfindahl, confrontando o número de patentes para cada classe tecnológica (IPC), com o número total de patentes de $i$ empresas para cada ano $t$. Como indicado por Hall (2005) esse indicador é ajustado $\left(N_{i t} / N_{i t}-1\right)$ para corrigir o problema de viés em observações com poucas patentes por ano. $\mathrm{O}$ indicador é formalmente definido como:

$\operatorname{TECHDIV}_{i t}=\frac{N_{i t}}{N}\left(1-\sum_{k=1}^{K}\left(\frac{N_{i t, k}}{N_{i t}}\right)^{2}\right)$

Onde $N_{i t}$ é o número total de patentes para a empresa $i$ no ano $t$, enquanto que $k$ representa a classe tecnológica (IPC) em que a empresa patenteou e $K$ é o número total de classes tecnológicas em que a empresa é ativa.

\subsection{Variáveis independentes}

Uma variável central neste trabalho é a oportunidade tecnológica. Para tanto, propõe-se a variável (OPPOR) para medir o aumento da taxa de atividade inovativa nas classes tecnológicas que a firma opera (MALERBA; ORSENIGO, 1993). Essa variável pressupõe que o conjunto de oportunidades para investimento e crescimento percebidas por empresários e administradores é específico de cada firma, como sugerem Penrose e Pitelis (2009).

Em geral as oportunidades de desenvolvimento tecnológico são multifacetadas, e a despeito da importância do seu conceito não é trivial a sua definição operacional e mensuração (KLEVORICK et al., 1995). Neste trabalho a variável OPPOR é calculada para cada firma como a taxa de crescimento geométrico ano a ano do número de patentes para cada classe tecnológica e foi inspirada na abordagem proposta por Patel e Pavitt (1998) e Corradini, Demirel, Battisti (2016). Para considerar a possibilidade de uma relação curvilínea, ou seja, o formato de "U" da variável oportunidade tecnológica, estima-se o seu termo ao quadrado (OPPOR²), que se espera apresentar um sinal negativo.

Para tratar dos efeitos de diferentes competências e capacitações das firmas, estima-se o seu estoque de conhecimento por meio do número de patentes acumulado em períodos prévios (KSTOCK). A construção dessa variável é originalmente inspirada na função de acumulação de Popp (2012), mas excluindo o componente de difusão, como proposto por Costantini e Mazzanti (2012). Da mesma forma, assim como convencionado pela literatura, essa variável considera uma taxa de depreciação de 15\% (HALL; JAFFE; TRAJTENBERG, 2005). Do mesmo modo, controlamos a heterogeneidade das firmas, tendo como fonte o banco de dados da Orbis. Nessa base, as firmas são classificadas pelo seu tamanho ${ }^{7}$, partindo de informações sobre a receita operacional, o total de ativos e o total de empregados, conforme tabela 1 a seguir.

${ }^{7}$ Originalmente: small, medium size, large e very large companies. 
Tabela 1 - Critérios para definição das categorias de tamanho das firmas.

\begin{tabular}{lccc}
\hline \multicolumn{1}{c}{ Tamanho das Firmas } & Receita operacional (US\$) & Total de ativos (US\$) & Total de empregados \\
\hline Pequenas & $\leq 1,3$ milhão & $\leq 2,6$ milhões & $\leq 15$ \\
Médio & $\geq 1,3$ milhão & $\geq 2,6$ milhões & $\geq 15$ \\
Médio-grande & $\geq 13$ milhões & $\geq 26$ milhões & $\geq 150$ \\
Grande & $\geq 130$ milhões & $\geq 260$ milhões & $\geq 1.000$ \\
\hline
\end{tabular}

Fonte: Elaboração do autor.

\subsection{Estimação do modelo}

$\mathrm{Na}$ análise proposta neste trabalho, a variável dependente é representada pela medida de diversificação tecnológica em que os valores oscilam no intervalo $I=(0,1)$. Esses dados não são normalmente distribuídos e sua natureza restrita a 0 e 1 pode levar a valores presumidos em uma regressão com mínimos quadrados ordinários. A alternativa de modelar o $\log$ da razão de chances (log-odds ratio) como uma função linear é inadequada, uma vez que não pode lidar com os casos em que a variável dependente é igual aos limites do intervalo de 0 e 1 . Da mesma forma, o ajustamento de valores extremos também é de difícil justificativa quando estes representam uma grande porcentagem dos dados (PAPKE; WOOLDRIDGE, 1996).

As variáveis fracionárias são observadas em muitos contextos econômicos, neste trabalho utiliza-se o modelo de resposta proposto por Papke e Wooldrige (1996), que sintetizam e expandem a literatura sobre modelos lineares generalizados (MLG) e quase-verossimilhança para obter métodos robustos para estimação e inferência com variáveis de resposta fracionária. Esse modelo assume a disponibilidade de uma sequência de observações, não normalmente distribuída $\left\{\left(x_{i}, y_{i}\right): i=1,2, \ldots, N\right\}$, onde $0 \leq y_{i} \leq 1$ e $N$ é o tamanho da amostra. A análise assintótica é realizada como $N \rightarrow \infty$ e a suposição mantida é que, para todo i,

$E\left(y_{i} \mid x_{i}\right)=G\left(x_{i} \beta\right)$

Onde $G($.$) é uma função de distribuição cumulativa, como a função logística G(z)=$ $\exp (z) /(1+\exp (z))$ que restringe $\mathrm{z}$ ao intervalo limitado aberto $I=(0,1)$. Desta forma, é possível maximizar a função de probabilidade logarítmica de Bernoulli expressa como:

$l_{i}(\beta)=y_{i} \log \left[G\left(x_{i} \beta\right)\right]+\left(1-y_{1}\right) \log \left[1-G\left(x_{i} \beta\right)\right]$

para obter um estimador de máxima quase-verossimilhança de $\beta$ que é consistente independentemente da distribuição $y_{i}$ condicional a $x_{i}$ usando regressões logit ou probit. Assim, dada a estrutura não-balanceada dos dados e o número limitado de observações por painel, os dados foram organizados pressupondo o potencial dos erros-padrão robustos em explicar heterogeneidades e a dependência estatística das séries ao longo do tempo.

Em análises adicionais, também se estima um modelo logístico de máxima verossimilhança padrão. Para tanto, converte-se a variável dependente em uma variável binária (TECHDIV_B), que é igual a 1 para todas as observações onde TECHDIV é maior que zero.

\section{$5 \quad$ Resultados empíricos}

Tendo como ponto de partida a identificação de depósitos de patentes com campos técnicos relacionados a tecnologias de mitigação das mudanças climáticas, o trabalho mapeia as 436 firmas que possuem uma sistemática e coerente atividade inventiva nessa área. Essas firmas representam um conjunto heterogêneo 
em diversos aspectos. Estão geograficamente distribuídas em 23 países, a destacar o Japão com 30\% do total, Estados Unidos com $26 \%$ e Alemanha com $16 \%$ e suas atividades econômicas principais estão distribuídas em 116 áreas, de acordo com a classificação estatística de atividades econômicas da Comunidade Europeia (NACE Rev. 2.0).

A Tabela 2 revela que a grande maioria das firmas são de grande porte $(76,6 \%)$, as firmas médias representam $7,8 \%$ e as pequenas constituem $15,6 \%$ do total. Os resultados também revelam que as firmas com tamanho médio são as mais diversificadas, seguida pelas firmas de grande porte. As firmas de tamanho pequeno e médio-grande são menos diversificadas. Por outro lado, as firmas de tamanho médio-grande são as que mais capturam atuam em ambiente com alta taxa de atividade inovativa, enquanto que essa taxa é menor nas firmas de grande porte. Uma possível explicação para esse resultado é a maior facilidade de firmas médias possuem de ingressar em áreas tecnológicas em rápida transformação em relação as grandes firmas.

Tabela 2 - Distribuição das firmas por tamanho, diversificação e oportunidade tecnológica.

\begin{tabular}{lccc}
\hline Tamanho das Firmas & TechDiv & Oppor & Número de Firmas \\
\hline Pequenas & 0,4615 & 0,5050 & 68 \\
Médio & 0,5875 & 0,5040 & 13 \\
Médio-grande & 0,3920 & 0,6091 & 21 \\
Grande & 0,5593 & 0,4986 & 334 \\
\hline
\end{tabular}

Fonte: Elaboração do autor.

A Tabela 3 descreve as principais estatísticas descritivas das variáveis que são utilizadas no modelo de regressão. Esses resultados mostram que existe uma importante diferença entre as firmas no que se refere ao seu nível de diversificação tecnológica, que também refletem o grande escopo de áreas técnicas analisadas. Do mesmo modo, as firmas se diferenciam no seu nível de oportunidades tecnológicas, ou seja, na sua capacidade de capturar essas oportunidades entre tecnologias ao longo do tempo.

Tabela 3 - Estatísticas descritivas das variáveis utilizadas no modelo.

\begin{tabular}{llcrrr}
\hline \multicolumn{1}{c}{ Descrição } & Média & $\begin{array}{c}\text { Desvio } \\
\text { Padrão }\end{array}$ & Mínimo & Máximo \\
\hline TECHDIV & $\begin{array}{l}\text { Indicador de diversificação tecnológica - dispersão do } \\
\text { portfólio de patentes entre classes } \\
\text { Variável binária construída a partir do indicador } \\
\text { TECHDIV_B }\end{array}$ & 0,5400 & 0,2953 & 0 & 1 \\
OPPOR & $\begin{array}{l}\text { TECHDIV para estimação dos modelos logit. } \\
\begin{array}{l}\text { Condições de oportunidade - taxa de mudança setorial na } \\
\text { atividade de patenteamento }\end{array}\end{array}$ & $0,0,5040$ & 0,40678 & $-0,5075$ & 2,9644 \\
KSTOCK & $\begin{array}{l}\text { Estoque de conhecimento da firma - patentes } \\
\text { acumuladas pela firma em períodos prévios. }\end{array}$ & 3,7856 & 1,3807 & 0,5306 & 8,0559 \\
\hline
\end{tabular}

Fonte: Elaboração do autor.

Sobre as estimativas econométricas da relação entre diversificação e oportunidades tecnológicas, a Tabela 4 apresenta os seus resultados principais. A primeira e a segunda coluna apresentam a estimação do modelo fracionário e a terceira coluna o modelo logístico. Os exercícios econométricos iniciam com a inclusão das variáveis lineares e quadrática da oportunidade tecnológica para explicar a não-linearidade na relação entre as oportunidades e diversificação tecnológica, juntamente com as variáveis de controle, para em seguida incluir a variável estoque de conhecimento das firmas.

Nos modelos fracionários, as variáveis lineares e quadráticas da oportunidade tecnológica são estatisticamente significativas enquanto no modelo logístico padrão, apenas as variáveis lineares. De qualquer forma, em todas as especificações e em todos os modelos o coeficiente associado ao OPPOR é 
positivo e o coeficiente associado a $\mathrm{OPPOR}^{2}$ é negativo. Esses resultados representam uma importante evidência da presença de uma relação não linear em formato de "U" invertido entre as oportunidades tecnológicas e diversificação tecnológica.

Tabela 4 - Estimativa dos coeficientes das regressões logit fracionária e logit padrão.

\begin{tabular}{|c|c|c|c|c|}
\hline Variável dependente & TECHDIV & & & \\
\hline Modelo & Logit fracionária & & Logit & \\
\hline Variáveis independentes e de controle & $(1)$ & (2) & $(3)$ & (4) \\
\hline \multirow[t]{2}{*}{ OPPOR } & $0,9359 * * *$ & $0,7563 * * *$ & $3,0598 * * *$ & $2,8355 * * *$ \\
\hline & $(0,2046)$ & $(0,1898)$ & $(0,4490)$ & $(0,4977)$ \\
\hline \multirow[t]{2}{*}{ OPPOR $^{2}$} & $-0,5007 * * *$ & $-0,4049 * * *$ & $-0,6140$ & $-0,6194$ \\
\hline & $(0,1333)$ & $(0,1178)$ & $(0,4593)$ & $(0,4801)$ \\
\hline \multirow[t]{2}{*}{ KSTOCK } & & $0,0009^{* * *}$ & & $0,0094 * * *$ \\
\hline & & $(0,0001)$ & & $(0,0020)$ \\
\hline \multirow[t]{2}{*}{ Constante } & $-0,1009$ & $-0,1318 *$ & $1,1399 * * *$ & $0,7499 * * *$ \\
\hline & $(0,0685)$ & $(0,0688)$ & $(0,1198)$ & $(0,1685)$ \\
\hline Dummies períodos & $\mathrm{Sim}$ & Sim & Sim & $\operatorname{Sim}$ \\
\hline Dummies tamanho & Sim & Sim & Sim & Sim \\
\hline Número de firmas & 436 & 436 & 436 & 436 \\
\hline Número de observações & 1473 & 1473 & 1473 & 1443 \\
\hline Log pseudo-verossimilhança & $-801,28$ & 752,55 & $-448,44$ & $-391,40$ \\
\hline $\mathrm{AIC}$ & 1,0624 & 1,0485 & & \\
\hline
\end{tabular}

Fonte: Elaboração do autor. $* p<0,10 ; * * p<0,05 ; * * * p<0,01$. Erro-padrão robusto em parênteses.

Ainda que uma relação positiva entre oportunidade e diversificação tecnológica seja esperada, o formato "U" invertido sugere que em ambientes tecnológicos de alta instabilidade e complexidade, as firmas tendem a adotar estratégias de especialização, em detrimento da diversificação. Esse resultado vai de encontro a noção convencional de que esses contextos suscitam uma menor especialização das firmas para lidar com a resolução destas incertezas (TOH; KIM, 2013).

O comportamento em direção a especialização em ambientes com crescente instabilidade e complexidade foi evidenciado para empresas inovadoras de pequeno porte no Reino Unido (CORRADINI; DEMIREL; BATTISTI, 2016). No presente artigo, as análises realizadas a partir do portfólio das firmas mais ativas em tecnologias de mitigação das mudanças climáticas revelam que na presença destes ambientes a estratégia adotada é a especialização. Desta forma, a relação não linear em formato de "U" invertido sugere que a diversificação tecnológica, também nesse caso, é determinada por riscos e capacitações envolvidas na atividade inovativa. Como resultado, as firmas precisam lidar com uma tensão constante entre a necessidade de manutenção de uma base de conhecimentos coerente e a capacitação para distribuir seus investimentos em muitos campos tecnológicos. Corroborando com a literatura, a coerência dessa base de conhecimento é um requisito fundamental para qualquer estratégia de diversificação e aumenta a chance sucesso do investimento em diferentes áreas tecnológicas (CHIU et al., 2010; MILLER, 2006; QUINTANA-GARCÍA; BENAVIDES-VELASCO, 2008).

Uma característica importante das tecnologias de mitigação das mudanças climáticas é a sua vantagem potencial de exploração em escala internacional, já que os esforços controle das emissões envolvem as principais nações industrializadas e as economias em desenvolvimento (HALL; HELMERS, 2013; OCKWELL et al., 2010). Por outro lado, o aproveitamento dessas oportunidades requer capacitações tecnológicas avançadas, dada a complexidade da gestão dos conhecimentos, as novas combinações requeridas, bem como os problemas de coordenação entre tecnologias e distâncias geográficas (SOMAYA; TEECE, 2008; ZANDER, 1997). 
Esses requisitos corroboram a segunda evidência apresentada no artigo de que a diversificação tecnológica em tecnologias de mitigação das mudanças climáticas é positivamente afetada pelo tamanho dos estoques de conhecimento das firmas. Esses resultados confirmam a expectativa inicial e são significativos em todos os modelos analisados. Em outras palavras, firmas alcançam maiores níveis de diversificação nessas tecnologias pelo acúmulo de conhecimentos.

\section{Conclusões}

Este artigo teve como objetivo investigar se a diversificação das firmas em tecnologias de mitigação das mudanças climáticas é afetada por alterações no nível de oportunidades tecnológicas. Para tanto, foi constituído um banco de dados original com patentes relacionadas a tecnologias de mitigação das mudanças climáticas, possibilitando a análise de 120 grupos de tecnologias em todo mundo e a evolução do portfólio de patentes de 436 firmas, entre os anos 1990-2009.

As estimativas econométricas oferecem evidências empíricas originais sobre a natureza e determinantes da diversificação tecnológica no que se refere ao papel exercido por mudanças nos níveis de oportunidade tecnológica. Os resultados revelam uma relação não linear e com formato "U" invertido entre oportunidade e diversificação tecnológica, ou seja, ao ingressar em ambientes tecnológicos caracterizados pela alta instabilidade e complexidade, firmas tendem a adotar a estratégia de especialização, ao invés de da diversificação.

$\mathrm{O}$ artigo revela de forma abrangente o papel que o ambiente tecnológico exerce sobre a atividade inovativa das firmas, bem como o impacto que sobre o grau de diversificação tecnológica. Enquanto a busca por um maior grau de diversificação tecnológica é crítica em diversos aspectos, firmas ao lidar com ambientes em rápida transformação, tendem a adotar estratégias de especialização, direcionamento dos seus investimentos e reforço das áreas tecnológicas que já atua. As possíveis explicações para esse comportamento é a exigência de capacitações tecnológicas avançadas para exploração destas tecnologias, a necessidade de um amplo escopo de recursos e os problemas de coordenação entre tecnologias e distâncias geográficas. Esses achados vão ao encontro dos resultados que também mostram a diversificação tecnológica positivamente afetada pelos estoques de conhecimento. Em outras palavras, as estratégias de diversificação nessas tecnologias são adotadas por firmas com elevada capacidade de acumulação de conhecimentos. Novas pesquisas nesse tema devem ser orientadas a novas variáveis que expliquem a exploração tecnológica internacional dessas tecnologias e uma ampliação da caraterização das firmas. 


\section{Referências}

ANSOFF, I. Strategies for Diversification. Harvard Business Review, v. 35, n. 5, p. 113-124, 1957.

BERRY, C. H. Corporate Growth and Diversification. The Journal of Law \& Economics, v. 14, n. 2, p. 371-383, 1971.

BRESCHI, S.; LISSONI, F.; MALERBA, F. Knowledge-relatedness in firm technological diversification. Research Policy, v. 32, n. 1, p. 69-87, 2003.

CANTWELL, J. A.; GAMBARDELLA, A.; GRANSTRAND, O. Technological and corporate diversification. In: The Economics and Management of Technological Diversification. $1^{\mathrm{a}}$. ed. London: Routledge, 2004. p. 1-17.

CANTWELL, J.; FAI, F. Firms as the source of innovation and growth: the evolution of technological competence. Journal of Evolutionary Economics, v. 9, p. 331-366, 1999.

CHANG, K.-C. et al. Nonlinear Effect of Technological Diversification on the Corporate Patent Performance. Journal of Applied Sciences, v. 14, n. 3, p. 273-278, 2014.

CHEN, Y. S.; SHIH, C. Y.; CHANG, C. H. The effects of related and unrelated technological diversification on innovation performance and corporate growth in the Taiwan's semiconductor industry. Scientometrics, v. 92, n. 1, p. 117-134, 2012.

CHIU, Y. C. et al. Technological scope: Diversified or specialized. Scientometrics, v. 82, n. 1, p. 37-58, 2010.

CORRADINI, C.; BATTISTI, G.; DEMIRELY, P. Serial innovators in the UK: Does size matter? Industrial and Corporate Change, v. 25, n. 1, p. 23-47, 2016.

CORRADINI, C.; DEMIREL, P.; BATTISTI, G. Technological diversification within UK's small serial innovators. Small Business Economics, v. 47, n. 1, p. 163-177, 2016.

COSTANTINI, V.; MAZZANTI, M. On the green and innovative side of trade competitiveness? the impact of environmental policies and innovation on EU exports. Research Policy, v. 41, n. 1, p. 132-153, 2012.

DE RASSENFOSSE, G.; PALANGKARAYA, A.; WEBSTER, E. Why do patents facilitate trade in technology? Testing the disclosure and appropriation effects. Research Policy, v. 45, n. 7, p. 1326-1336, 2016.

DOSI, G.; NELSON, R. R.; WINTER, S. G. Introduction: The Nature and Dynamics of Organizational Capabilities. In: The Nature and Dynamics of Organizational Capabilities. $1^{\text {a }}$. ed. Oxford: Oxford University Press, 2001. p. 1-22.

GARCIA-VEGA, M. Does technological diversification promote innovation?: An empirical analysis for European firms. Research Policy, v. 35, n. 2, p. 230-246, 2006.

GEELS, F. W. Reconceptualising the co-evolution of firms-in-industries and their environments: Developing an inter-disciplinary Triple Embeddedness Framework. Research Policy, v. 43, n. 2, p. 261277, 2014. 
GORT, M. Diversification and Integration in American Industry. 1 ${ }^{\text {a }}$. ed. Princeton: Princeton University Press, 1962.

GRANSTRAND, O.; PATEL, P.; PAVITT, K. Multi-Technology Corporations: Why they have "distributed" rather than "distinctive core" competencies. California Management Review, v. 39, n. 4, p. 8-25, 1997.

HALL, B. A note on the bias in the herfindahl based on count data. Revue d'économie industrielle, v. 110 , p. 149-156, 2005.

HALL, B. H.; HELMERS, C. Innovation and diffusion of clean/green technology: Can patent commons help? Journal of Environmental Economics and Management, v. 66, n. 1, p. 33-51, 2013.

HALL, B. H.; JAFFE, A. B.; TRAJTENBERG, M. Market Value and Patent Citations. RAND Journal of Economics, v. 36, n. 1, p. 16-38, 2005.

HELFAT, C. E. et al. Dynamic capabilities : understanding strategic change in organizations. $1^{\text {a }}$. ed. Malden: Blackwell Publishing Ltd, 2007.

HENDERSON, R. M.; COCKBURN, I. Scale, scope and spillovers: the determinats of research productivity in drug discovery. Rand Journal of Economics, v. 27(1), n. 1, p. 32-59, 1996.

HODGSON, G. Competence and contract in the theory of the firm. Journal of Economic Behavior and Organization, v. 35, n. 2, , p. 179-201, 1998.

KAMIEN, M. I.; SCHWARTZ, N. L. Market structure and innovation: A survey. Journal of economic literature, v. 13, n. 1, p. 1-37, 1975.

KLEVORICK, A. K. et al. Policy in Technological Opportunities. Research in Economics, v. 24, p. 185$205,1995$.

LETEN, B.; BELDERBOS, R.; VAN LOOY, B. Technological diversification, coherence, and performance of firms. Journal of Product Innovation Management, v. 24, n. 6, p. 567-579, 2007.

LIN, C.; CHANG, C. C. The effect of technological diversification on organizational performance: An empirical study of S\&P 500 manufacturing firms. Technological Forecasting and Social Change, v. 90, n. PB, p. 575-586, 2015.

MALERBA, F.; ORSENIGO, L. Technological regimes and firm behaviour. Industrial and Corporate Change, v. 19, n. 1, p. 45-71, 1993.

MILLER, D. J. Technological diversity, related diversification, and firm performance. Strategic Management Journal, v. 27, n. 7, p. 601-619, 2006.

NELSON, R. R.; WINTER, S. G. An evolutionary theory of economic change. Cambridge: Belknap Press of Harvard University Press, 1982. v. 93

NOOTEBOOM, B. et al. Optimal cognitive distance and absorptive capacity. Research Policy, v. 36, n. 7, p. 1016-1034, 2007. 
OCKWELL, D. G. et al. Intellectual property rights and low carbon technology transfer: Conflicting discourses of diffusion and development. Global Environmental Change, v. 20, n. 4, p. 729-738, 2010.

OECD. OECD Science, Technology and Industry Outlook 2014. Paris: OECD Publishing, 2014.

PAPKE, L. E.; WOOLDRIDGE, J. M. Econometric methods for fractional response variables with an application to 401 (K) plan participation rates. Journal of Applied Econometrics, v. 11, n. 6, p. 619-632, 1996.

PATEL, P.; PAVITT, K. The technological competencies of the world's largest firms: Complex and pathdependent, but not much variety. Research Policy, v. 26, n. 2, p. 141-156, 1997.

PATEL, P.; PAVITT, K. The Wide (and Increasing) Spread of Technological Competencies in the World's Largest Firms: A Challenge to Conventional Wisdom. In: The Dynamic Firm: The Role of Technology, Strategy, Organization, and Regions. 1 ${ }^{\text {a }}$. ed. Oxford: Oxford University Press, 1998. p. 192-213.

PAVITT, K. Technologies, Products and Organization in the Innovating Firm: What Adam Smith Tells Us and Joseph Schumpeter Doesn't. Industrial and Corporate Change, p. 433-452, 1998.

PENROSE, E. T.; PITELIS, C. N. The theory of the growth of the firm. $4^{\text {a }}$. ed. Oxford: Oxford University Press, 2009.

PITTS, R. A.; HOPKINS, H. D. Firm Diversity: Conceptualization and Measurement. The Academy of Management Review, v. 7, n. 4, p. 620-629, 1982.

PODOLNY, J. M.; STUART, T. E. A Role-Based Ecology of Technological Change. American Journal of Sociology, v. 100, n. 5, p. 1224-1260, 1995.

POPP, D. Induced Innovation and Energy Prices. American Economic Review, v. 92, n. 1, p. 160-180, 2012.

QUINTANA-GARCÍA, C.; BENAVIDES-VELASCO, C. A. Innovative competence, exploration and exploitation: The influence of technological diversification. Research Policy, v. 37, n. 3, p. 492-507, 2008.

RAMANUJAM, V.; VARADARAJAN, P. Research on Corporate Diversification: A Synthesis. Strategic Management Journal, v. 10, n. 6, p. 523-551, 1989.

SCHERER, F. M. Inter-industry technology flows in the United States. Research Policy, v. 11, n. 4, p. 227-245, 1982.

SCOTT, J. T. Purposive Diversification and Economic Performance. $1^{\text {a }}$. ed. Cambridge: Cambridge University Press, 1993.

SOMAYA, D.; TEECE, D. J. Patents, Licensing, and Entrepreneurship: Effectuating Innovation in Multiinvention Contexts. In: The transfer and licensing of know-how and intellectual property: understanding the multinational enterprise in the modern world. $1^{\text {a }}$. ed. Singapore; London: World Scientific Publishing Co. Pte. Ltd., 2008. p. 287-314.

TEECE, D. J. Economies of scope and the scope of the enterprise. Journal of Economic Behavior \& Organization, v. 1, n. 3, p. 223-247, set. 1980. 
TEECE, D. J. Towards an economic theory of the multiproduct firm. Journal of Economic Behavior \& Organization, v. 0, n. 0, p. 39-62, mar. 1982.

TEECE, D. J. et al. Theory corporate coherence and evidence. Journal of Economic Behavior and Organization, v. 23, p. 1-30, 1994.

TEECE, D.; PISANO, G.; SHUEN, A. Dynamic capabilities and strategic management. Strategic management journal, v. 18, n. 7, p. 509-533, 1997.

TOH, P. K.; KIM, T. Why put all your eggs in one basket? A competition-based view of how technological uncertainty affects a firm's technological specialization. Organization Science, v. 24, n. 4, p. 1214-1236, 2013.

TORRISI, S.; GRANSTRAND, O. Technological and business diversification: A survey of theories and empirical evidence. In: The Economics and Management of Technological Diversification. $1^{\text {a }}$. ed. London: Routledge, 2004. p. 21-68.

ZANDER, I. Technological diversification in the multinational corporation-historical evolution and future prospects. Research Policy, v. 26, n. 2, p. 209-227, 1997. 
Anexo - Hierarquia dos grupos de tecnologias.

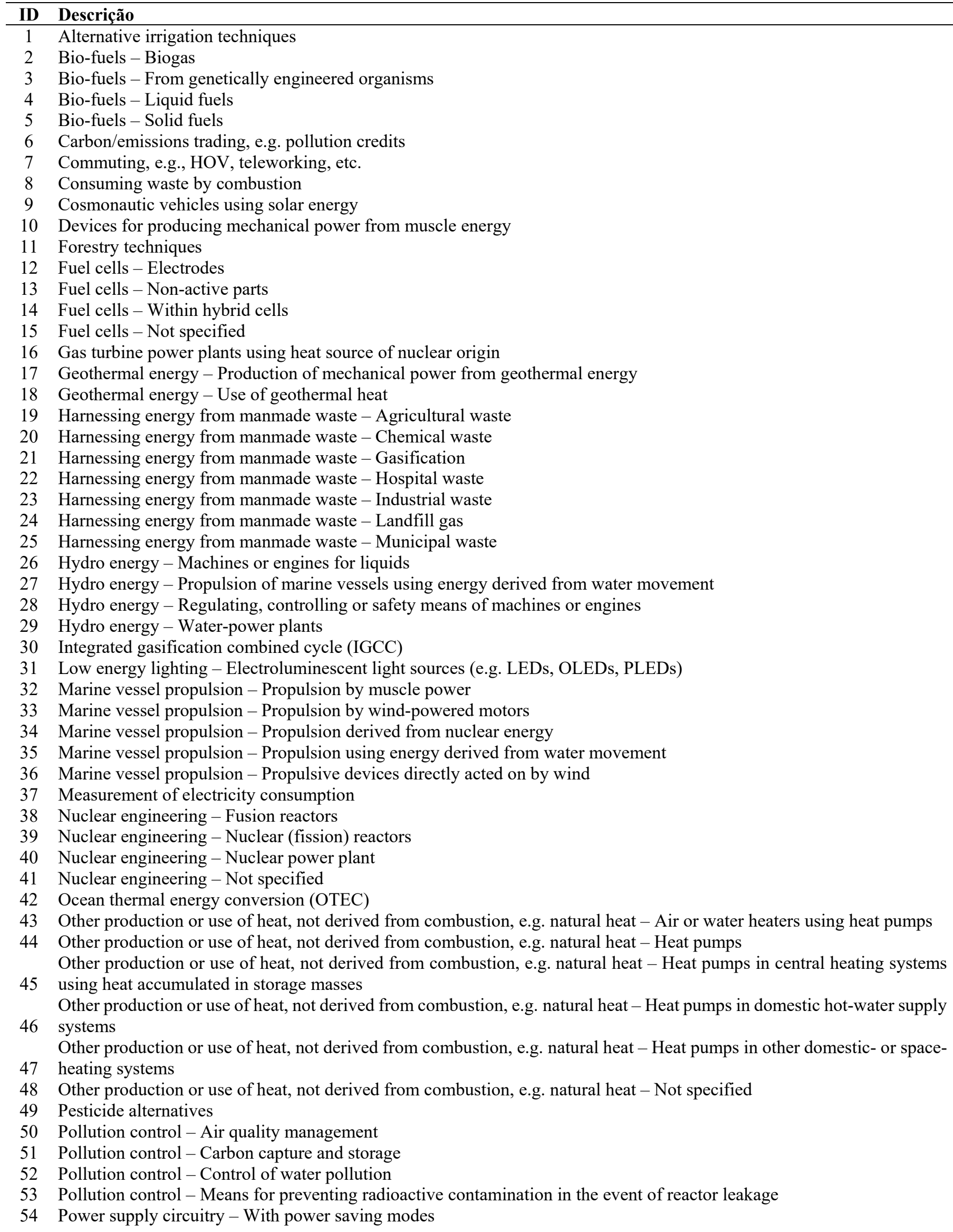




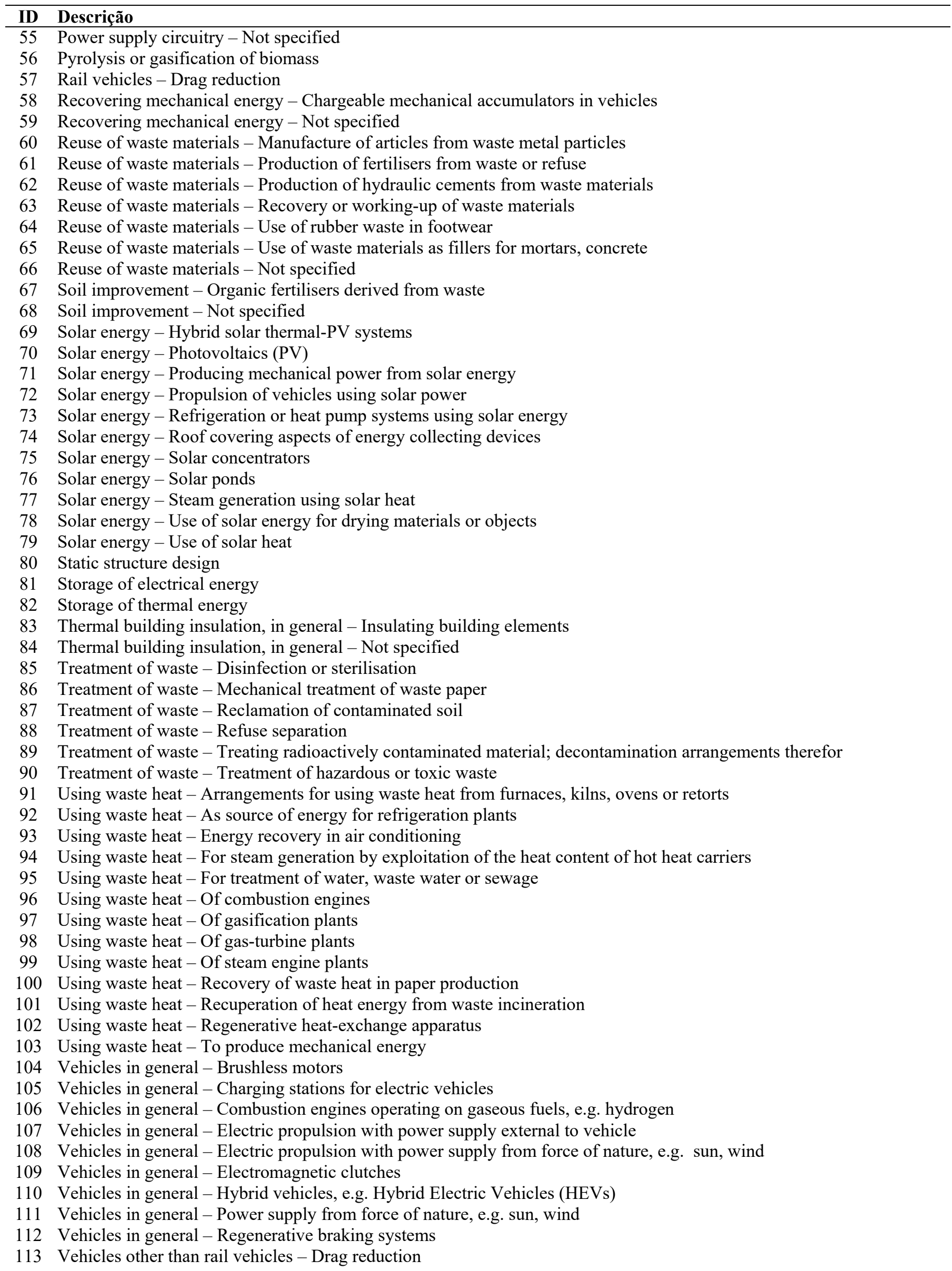




\begin{tabular}{cl}
\hline ID & Descrição \\
\hline 114 & Vehicles other than rail vehicles - Human-powered vehicle \\
115 & Waste disposal \\
116 & Wind energy - Propulsion of marine vessels by wind-powered motors \\
117 & Wind energy - Propulsion of vehicles using wind power \\
118 & Wind energy - Structural aspects of wind turbines \\
119 & Wind energy - Structural association of electric generator with mechanical driving motor \\
120 & Wind energy - Not specified \\
\hline
\end{tabular}

